\title{
The Futility of Observing Stimulated Gamma-Ray Emission from a Long-Lived Isomeric State.
}

\author{
G. C. BALDWin and J. C. Solem
}

Theoretical Division, Los Alamos National Laboratory - Los Alamos, NM 87545, USA

(ricevuto l'8 Settembre 1994; approvato il 20 Ottobre 1994)

\begin{abstract}
Summary. - We reveal the fallacies in recent gamma-ray laser schemes that propose to obtain gain by stimulating transitions from a long-lived upper to a short-lived lower nuclear state. We point out the errors in recent derivations of the stimulated-emission coefficients, derive the correct formulae, and submit an alternative explanation for an experiment purported to observe stimulated emission from a long-lived isomer.
\end{abstract}

PACS 76.80 - Mössbauer effect; other $\gamma$-ray spectroscopy.

We have recently become aware of publications in this journal [1-3] whose authors dispute the general consensus that stimulated emission of recoilless gamma-radiation from a long-lived nuclide is exceedingly difficult to observe [4]. These papers have raised hopes that an easy solution to the problem of creating a gamma-ray laser [5] is to use a long-lived isomer as the active material. The purpose of this paper is to explain the futility of such an approach.

Greaves et al. [1] present what they apparently believe is a new concept, with a supporting analysis. They incorrectly conclude that stimulated-emission gain is possible in a transition from a long-lived state to a short-lived excited state, because then, «... the line width of the radiation is totally determined by the breadth of the lower excited state[1]». They believe that inhomogeneous broadening is of no concern when the line breadth is dominated by the homogeneous broadening from lower-state decay. That is true, but they overlook the extremely small radiation width of the long-lived state.

Kamenov [3] claims that the commonly accepted formula for the stimulatedemission cross-section [4] applies only to transitions to the ground state of a nucleus (Mössbauer emission). For transitions between excited states, he asserts, the formula is not only incorrect, but underestimates the true cross-section by many orders of magnitude. He denies the existence of homogeneous line broadening. 
We now present the correct analysis and identify the flaw in Kamenov's treatment. The radiative lifetime of a state is given by

$$
\tau_{\mathrm{r}}=(1+\alpha) \tau,
$$

where $\tau$ is the natural lifetime and $\alpha$ is the ratio of electron-to-photon emission rates (internal conversion coefficient). Consider the low-lying states of ${ }^{125} \mathrm{Te}$, which Kamenov proposes to use as a GRASER: 1) level $m$, the $144.73 \mathrm{keV}$ state with $\alpha \simeq$ $\simeq 352$ and $\tau_{\mathrm{r}} \simeq 56 \mathrm{y} ; 2$ ) level $n$, the $35.46 \mathrm{keV}$ state with $\alpha \simeq 12.5$ and $\tau_{\mathrm{r}} \simeq 19.7 \mathrm{~ns}$; and 3) level $o$, the ground state. Place the 3 states of ${ }^{125} \mathrm{Te}$ in a cavity with walls at a uniform high temperature so that the population of nuclides is bathed in thermal radiation with the frequency spectrum given by Planck's formula

$$
\rho(v)=\frac{8 \pi h v}{c^{3}} \frac{1}{\exp [h v / k T]-1},
$$

where $v$ is the photon frequency, $h$ is Planck's constant, $c$ is the speed of light, $k$ is Boltzmann's constant, and $T$ is the temperature. Although equilibrium is actually transient because of the spontaneous decay by internal conversion, it is an adequate approximation to assume that thermal equilibrium can be established; the respective probabilities of occupancy of the three states will then be given by their Boltzmann factors,

$$
W_{i}=g_{i} \exp \left[E_{i} / k T\right],
$$

where the $E_{i}$ and $g_{i}$ are the respective energies and statistical weights of the states. As a result of balance between the downward (spontaneous and stimulated) and upward (resonant absorption) allowed transitions among all pairs of states, we have the relations

$$
\left\{\begin{array}{l}
\left(B_{m n} \rho\left(v_{m n}\right)+A_{m n}\right) W_{m}=B_{n m} \rho\left(\nu_{m n}\right) W_{n}, \\
\left(B_{n o} \rho\left(v_{n o}\right)+A_{n o}\right) W_{n}=B_{o n} \rho\left(v_{n o}\right) W_{o},
\end{array}\right.
$$

where $A$ and $B$ are the Einstein coefficients, the subscripts refer to the levels of the transition, and the order of the subscripts indicates the direction.

Invoking the Einstein relations of frequency to transition energy, consistency demands that

$$
\left\{\begin{array}{l}
B_{m n}=\left(\frac{g_{n}}{g_{m}}\right) B_{n m}=A_{m n}\left(\frac{c^{3}}{8 \pi h \nu_{m n}^{3}}\right), \\
B_{n o}=\left(\frac{g_{0}}{g_{n}}\right) B_{o n}=A_{n o}\left(\frac{c^{3}}{8 \pi h \nu_{n o}^{3}}\right),
\end{array}\right.
$$

from which we obtain the ratio of the stimulated-emission coefficients,

$$
\frac{B_{m n}}{B_{n o}}=\frac{A_{m n} \nu_{n o}^{3}}{A_{n o} \nu_{m n}^{3}} .
$$


For the nuclide ${ }^{125} \mathrm{Te}$, we find that the ratio of the stimulated-emission coefficients is

$$
\frac{B_{m n}}{B_{n o}} \simeq \frac{(56 \mathrm{y})^{-1}}{(19.3 \mathrm{~ns})^{-1}} \cdot \frac{(35.46 \mathrm{keV})^{3}}{(109.27 \mathrm{keV})^{3}} \simeq 3.8 \cdot 10^{-19} .
$$

So stimulated emission is a miniscule effect in this or in any other nuclide between a very-long-lived upper state and a very-short-lived lower state.

Here is Kamenov's error. In his derivation for transitions between excited states, instead of the second equation as given in eqs. (4), Kamenov [3] writes a single equation

$$
\mathrm{d} W_{m n}=\left(B_{m n} \rho(v)+\left(-A_{n o}\right)\right) \mathrm{d} t
$$

and asserts that, «for a time $\mathrm{d}$ t, either the transition $n \rightarrow m$ can occur or $n$ can decay spontaneously $(*)$ ". This is wrong; it overlooks the stimulated $n \leftrightarrow 0$ transitions. The Einstein treatment applies to a large population of similar nuclei, in which both kinds of transitions would be occurring concurrently between both pairs of states.

Alpatov et al. [6] have observed a peak in the measured pulse height spectrum of ${ }^{125 m} \mathrm{Te}$ at the channel corresponding to twice the $109.27 \mathrm{keV}$ transition energy. They correctly interpret this as the coincident arrival of two photons at their detector, both of which were detected by photoelectric rather than Compton effect. But they incorrectly assume, with support from Skorobogatov and Kamenov, that this is the result of stimulated emission in their source. Kamenov [3] and Skorobogatov [2] support this claim by the fallacious reasoning described above. It is well known to laser physicists that stimulated and stimulating photons are not emitted in time coincidence and so do not arrive at and cannot be registered simultaneously by a detector. We believe the double-energy pulses are chance coincidences of incoherent, spontaneously emitted photons. Alpatov et al. apparently did not consider this explanation; they do not report the source strength, the resolving time of their detector nor the counting rate, nor do they even make an estimate of the chance coincidence rate.

The relative rates of spontaneous downward, stimulated upward and stimulated downward transitions must differ by just the amount that maintains a Boltzmann distribution of populations in a bath of thermal radiation. The rate of spontaneous decay of level $m$ is very low; therefore, the stimulated-emission rates to and from this level must be correspondingly low.

Although population inversion is inherent in a three-level-cascade nuclear system whenever the lifetime of the upper state exceeds that of the lower, the cross-section for stimulated emission is correspondingly small. If the ratio of lifetimes is large, stimulated emission will be unobservable; the experiment that is claimed to demonstrate it has been misinterpreted.

Like all researchers who have worked in this area, we recognize the profound effect an operational gamma-ray laser would have on science and society. It is important that time and resources not be wasted and misconceptions not be propagated. Therefore, we discourage any attempts to design GRASERs based on nuclides having a three-level cascade in which the lifetime of the upper state greatly exceeds that of the lower state of the transition.

(*) Emphasis ours; italics are Kamenov's. 
We thank Prof. V. I. Kirischuk for calling our attention to the referenced papers of Alpatov et al. and Kamenov, and for discussions in correspondence regarding them.

\section{REFERENCES}

[1] E. D. Greaves, L. Sajo-Bohus and H. Groening: Nuovo Cimento D, 9, 1303 (1987).

[2] G. A. Skorobogatov: Nuovo Cimento D, 12, 793 (1990).

[3] P. S. Kamenov: Nuovo Cimento D, 13, 1369 (1991); also C. R. Acad. Bulg. Sci., 41, 55 (1988).

[4] G. C. Baldwin, J. C. Solem and V. I. Gol'danskir: Rev. Mod. Phys., 53, 587 (1981).

[5] V. I. KIRISCHUK: Institute for Nuclear Research, Ukraine, private communication.

[6] V. G. Alpatov, G. E. Bizina, A. V. Davydov, B. E. Dzevitskil, G. R. Kartaschov, M. M. Korotkov, G. V. Kostina, A. A. Sadovski and G. A. Skorobogatov: Izv. Akad. Nauk. USSR, Ser. Fiz., 50, 2013 (1986). 